\title{
¿Qué tan derecho es el derecho a la educación en México?
}

\author{
Raquel Ahuja Sánchez
}

\begin{abstract}
Resumen
Este artículo aborda el significado y los alcances de la educación como un derecho humano, para lo cual se exponen algunos conceptos centrales como son los principios de los derechos humanos y las dimensiones del derecho a la educación. Desde ese paradigma, presenta un breve panorama de la educación obligatoria en México, y se centra en las condiciones escolares que, de acuerdo a una evaluación realizada por el Instituto Nacional para la Evaluación de la Educación (INEE) a fines de 2014, presentan las escuelas primarias del país. Se plantea como conclusión la necesidad de que todas las escuelas de México cuenten con un conjunto irreductible de condiciones que garanticen un mínimo de oportunidades de aprendizaje para todos los estudiantes.
\end{abstract}

Palabras clave: derecho a la educación, condiciones escolares, escuelas primarias, desigualdad educativa.

\section{How Right Is the Right to Education in México?}

\begin{abstract}
This article approaches the meaning and the reaches of education as a human right. For this purpose we will be touching central principles such as human rights and the right to educationon. From this paradigm we will present a brief panorama of obligatory education in México, focusing on elementary school conditions as stated in the 2014 Report on Education Evaluation by the National Institute for the Evaluation of Education. We propose as conclusion the need to enforce all Mexican schools have an irreducible set of conditions that guarantee a minimum of learning opportunities for all students.
\end{abstract}

Key words: right to education, school conditions, elementary schools, educational inequality. 


\section{Raquel Ahuja Sánchez}

raquel.ahuja@gmail.com

Es Licenciada en Psicología por el Instituto Tecnológico y de Estudios Superiores de Occidente (ITESO), estudió la maestría en Psicología general experimental con énfasis en Salud en la Facultad de Psicología de la Universidad Nacional Autónoma de México (UNAM) y cursó un año del Doctorado en Derechos Humanos en la Universidad Nacional de Educación a Distancia (UNED) de España. En 1992 inició como investigadora y evaluadora en el campo de la educación, habiendo trabajado en el Centro de Estudios Educativos y en el Instituto de Investigaciones Multidisciplinarias en Educación de la Universidad Iberoamericana. A partir del 2000 se incorporó al servicio público ocupando diversos cargos directivos dentro de la Secretaría de Educación Pública. Actualmente trabaja en el Instituto Nacional para la Evaluación de la Educación (INEE), desde el 2012, donde ocupa la Dirección General de Evaluación de la Oferta Educativa.

\section{La educación como un derecho humano}

La reforma al artículo $3^{\circ}$ constitucional, que se llevó a cabo en 2013, introdujo el reconocimiento de la educación de calidad como un derecho humano que, como tal, debe cumplir con los cuatro principios de los derechos humanos: universalidad, indivisibilidad, interdependencia y progresividad. Si bien este artículo no tiene la intención de profundizar en aspectos jurídicos, consideramos importante recordar brevemente su significado.

La universalidad se refiere a que, al ser inherentes al ser humano, los derechos deben garantizarse para todos, sin distinciones de ningún tipo (credo, etnia, ideología, género, etcétera), ya que todas las personas son iguales en dignidad y derechos; la indivisibilidad alude a que no pueden protegerse unos derechos y otros no, pues los derechos humanos son todos derechos, no pueden priorizarse o jerarquizarse; la interdependencia refiere a la relación que existe entre los derechos humanos, por lo que de afectarse uno, se impacta sobre otros derechos (en el caso de la educación, el cual se considera un derecho clave o habilitante (Latapí, 2009), su incumplimiento afecta el acceso a otros derechos como al trabajo, la alimentación, la salud y la vivienda); por

1. Existe múltiple literatura sobre este tema, en la bibliografía se incluyen dos referencias con acceso digital tanto para una lectura ligera sobre el tema (texto de la CNDH) como para una más especializada (Vázquez y Serrano, s/f). último, la progresividad significa que cada vez deben ofrecerse más y mejores condiciones para el ejercicio de los derechos, y en ningún caso debe haber regresividad o reversibilidad. ${ }^{1}$

Visto así, el derecho a la educación es para todas las personas, independientemente de su contexto o condición, y con su cumplimiento deben protegerse también otros 


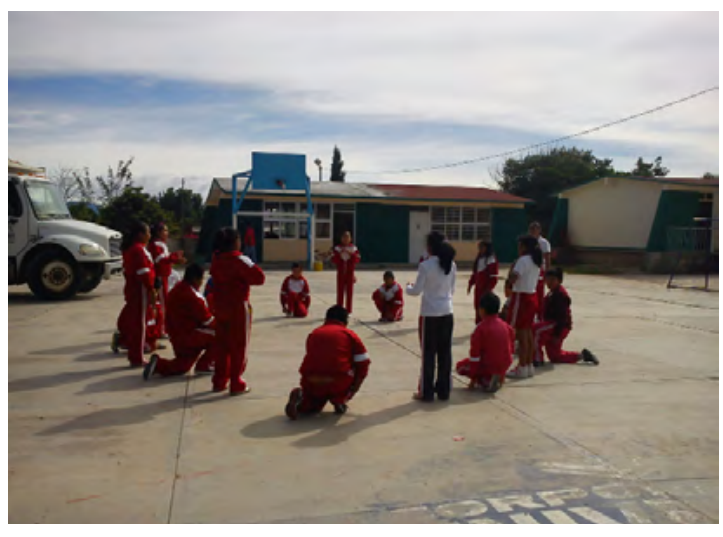

derechos. Como en cualquier derecho humano, es el Estado el responsable de promover, respetar, proteger y garantizar su cumplimiento, esto es, diseñar las políticas públicas y acciones para hacerlos efectivos.

¿Pero qué significa el derecho a la educación? El derecho a la educación no significa solamente el derecho a ir a la escuela -que es la institución del Estado mediante la cual se ofrece educación a la población- ya que si así fuera bastaría con que se dispusieran escuelas suficientes para todos. El derecho a la educación significa tanto el acceso a la escuela como su permanencia en ella y, sobre todo, el derecho a aprender y adquirir las competencias necesarias para que se alcancen las finalidades sociales de la educación. Esto implica que la educación debe tener ciertas características.

La primera relatora especial de las Naciones Unidas para el derecho a la educación, Katarina Tomasevski (2004), definió cuatro dimensiones del derecho a la educación, las cuales se conocen como el esquema de las 4 A: asequibilidad, accesibilidad, aceptabilidad y adaptabilidad. La asequibilidad se refiere a la disponibilidad de servicios educativos, que es el nivel más básico para garantizar el derecho a la educación; la accesibili-

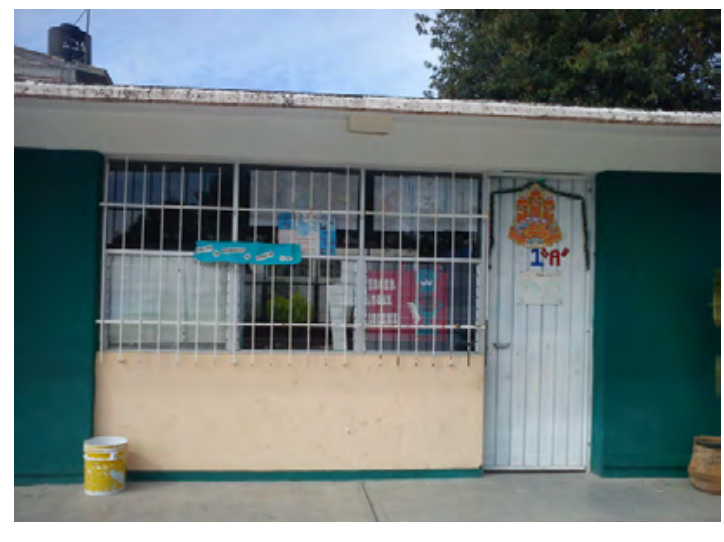
dad significa el acceso efectivo a las escuelas, es decir, que no haya barreras de ningún tipo que impidan que alguien llegue y transite por la escuela; la aceptabilidad cualifica los servicios educativos, al señalar que éstos deben cumplir con determinados estándares de calidad (como maestros con formación adecuada, materiales educativos pertinentes, escuelas con infraestructura suficiente y que

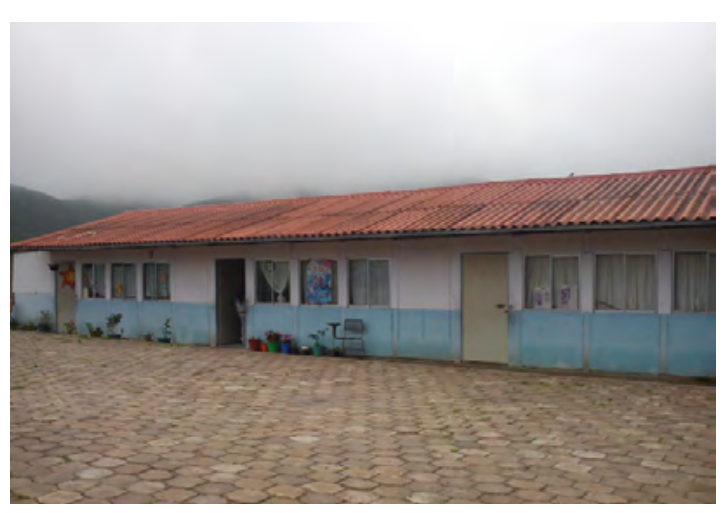
brinde seguridad a los estudiantes, etcétera); y, por último, la adaptabilidad, significa que los servicios educativos deben adaptarse a las características de la población y no al revés, es decir, tomar las medidas necesarias para atender con pertinencia a estudiantes migrantes, indígenas, a quienes no hablan la lengua de instrucción, a quienes presentan alguna discapacidad, etcétera. 
2. En 2012 se declaró obligatoria la educación media superior en el país.

3. La tasa de matriculación es un indicador que se refiere al porcentaje de niños, niñas y adolescentes de cada grupo de edad que está matriculado en la escuela, independientemente del nivel educativo y grado.

4. El avance regular es un indicador que considera los niños, niñas y adolescentes matriculados en el grado escolar ideal de acuerdo a su edad o en uno inferior o uno superior.

5. Muestra de ello es que, en tanto a nivel nacional, la escolaridad de la población de 15 años y más es de 9.4 años, para ese mismo grupo de edad en la población jornalera agrícola migrante es de 4.5, para la población hablante de una lengua indígena de 5.7 y para las personas con discapacidad de 5.1 (INEE, 2016b, 2017b y 2017c).

6. El nivel educativo de primaria es el que tiene el mayor número de escuelas, docentes y estudiantes del sistema educativo mexicano

Fuente: INEE (2017a).

\section{Breve panorama del derecho a la educación en México}

En México, la educación se ofrece en los siguientes tipos y niveles educativos.

El sistema educativo mexicano es uno de los más grandes del mundo, con más de 30 millones de estudiantes, 1.5 millones de docentes y poco más de 240 mil escuelas o planteles en la educación obligatoria, esto es, en los tipos básico y medio superior² (INEE, 2017a). Con estas cifras es posible ver que hemos avanza-

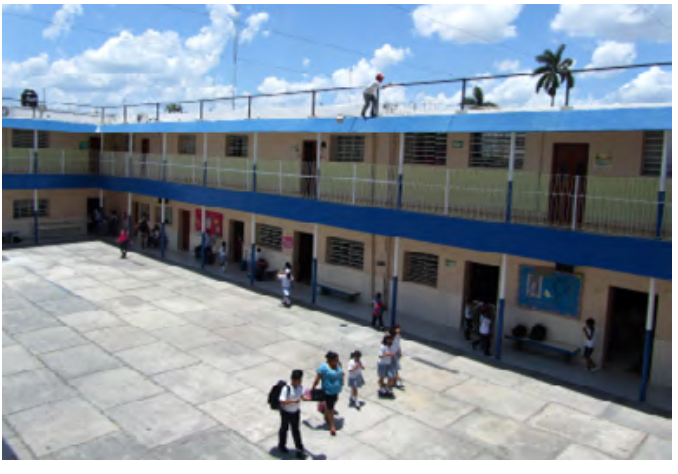
do en asequibilidad y accesibilidad de la educación obligatoria, sobre todo en educación básica. Las cifras más recientes disponibles (agosto de 2017), advierten que las tasas de matriculación ${ }^{3}$ a nivel nacional son superiores al 80\% para los niños y niñas de 3 a 5 años, de 6 a 11 y de 12 a 14, y de 65\% para los de 15 a 17 años; en estos grupos de edad el porcentaje de estudiantes que tiene un avance regular ${ }^{4}$ es superior a 92\% (INEE, 2016a). Aunque los datos son alentadores, como es sabido, la menor cobertura educativa se tiene en las poblaciones en situación de vulnerabilidad social: migrantes, indígenas y personas con discapacidad. ${ }^{5}$

Donde, sin duda, tenemos los mayores retos -y que es en lo que se pretende enfocar lo que resta de este artículo-, es en la aceptabilidad y la adaptabilidad de la educación, es decir, en la forma como operan y funcionan las escuelas.

\section{Condiciones escolares de las escuelas primarias ${ }^{6}$}

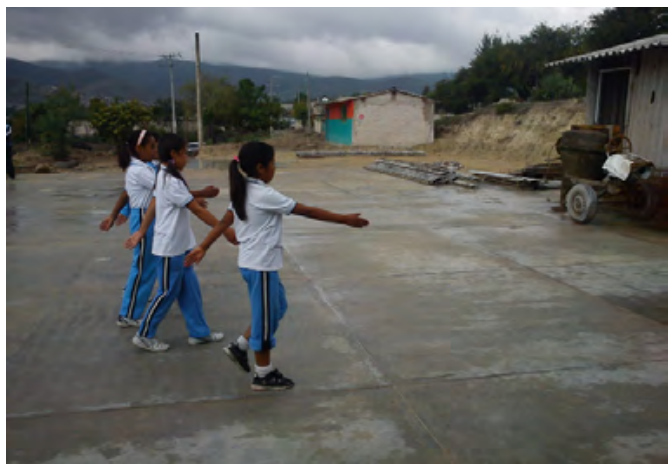

Cuando hablamos de condiciones escolares probablemente pensamos en equipamiento como computadoras, pizarrones electrónicos, materiales multimedia y espacios escolares "de primera": laboratorios equipados, biblioteca con acervos diversos, auditorios, canchas para practicar múltiples deportes; esto es porque consideramos que otras condiciones básicas "están dadas". Pero probablemente sorprenda a muchos saber que la mitad de las escuelas primarias del país tienen menos de seis maestros, es decir, no hay maestros para atender en grupos separados a estudiantes de un mismo grado, es más, una de cada ocho escuelas primarias generales o indígenas del país sólo tienen un docente, que es el maestro de todos los estudiantes de los seis grados y además el director de la escuela - 
7. Esto no necesariamente es así por insuficiencia de docentes, pero sí porque se anteponen criterios de eficiencia (número de estudiantes por docente) a los pedagógicos y de justicia social. este último dato no considera las escuelas de modalidad comunitaria, que son en su gran mayoría unitarias- (INEE, 2016a). ${ }^{7}$

\section{Esquema 1. Estructura del Sistema Educativo Nacion}

$-\frac{\text { Principales cifras }}{\text { Ciclo escolar 2015-2016 }}$

\section{Sistema Educativo Nacional}

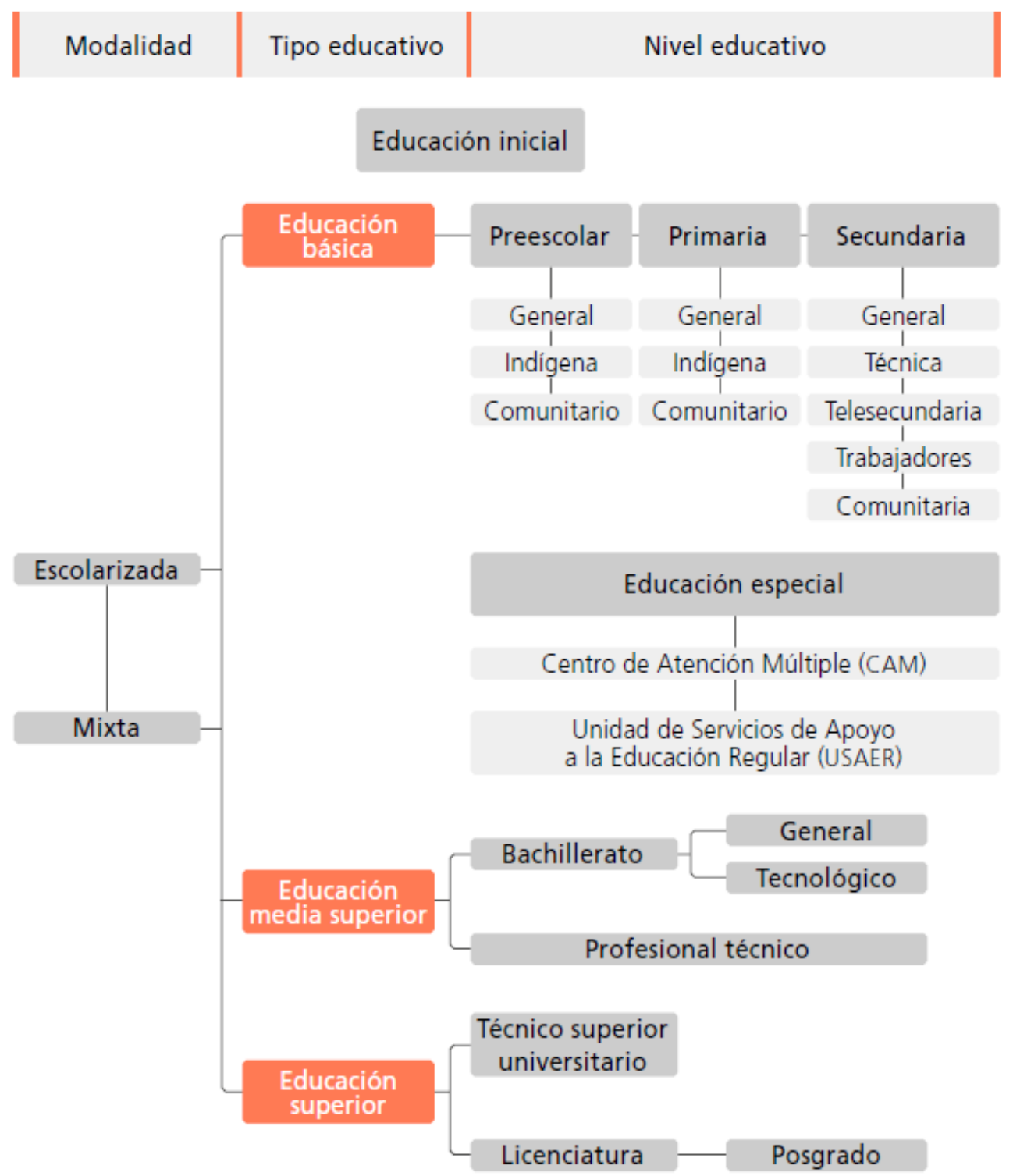


8. Para las poblaciones indígenas es un derecho humano recibir educación en su propia lengua.

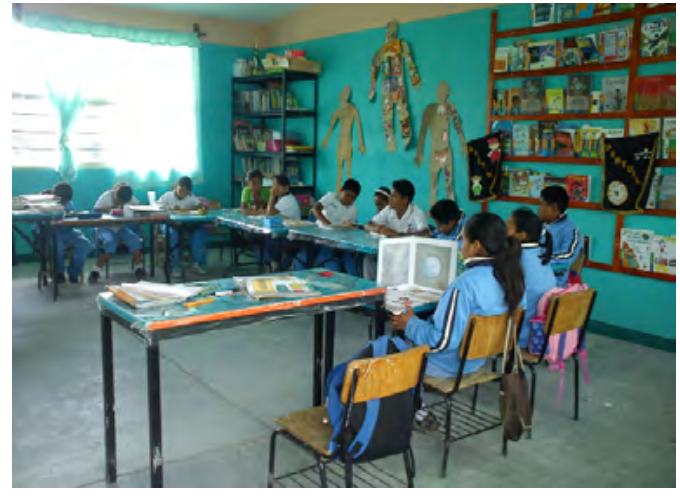

Otros datos que reflejan las condiciones en que operan las escuelas primarias en México son los siguientes: el $45 \%$ de las escuelas tiene los servicios básicos de agua, luz y drenaje; al 13.7\% de escuelas les faltan aulas para atender a todos los grupos; el 18.7\% no tiene tazas sanitarias para uso exclusivo de estudiantes. A uno de cada cuatro grupos de los últimos tres grados de primaria, les falta pizarrón en su salón de clases o el que tienen no funciona adecuadamente; poco más de un tercio de escuelas cuentan con libreros o estantes adecuados para guardar y exponer los materiales de la biblioteca escolar; en menos de una de cada cuatro escuelas hay acceso a internet para todos los miembros de la comunidad escolar; sólo uno de cada tres docentes que imparten la asignatura de lengua indígena, cuentan con el libro para el maestro de esa asignatura, y sólo en uno de cada cuatro grupos de estudiantes de $4^{\circ}, 5^{\circ}$ y $6^{\circ}$ grado que cursaban esta asignatura los estudiantes recibieron el libro de texto (INEE, 2016c, 2016d y 2016e). ${ }^{8}$

Lo anterior ocurre porque no se han distribuido de la misma manera los recursos, porque contrario a un principio de equidad que llevaría a que el Estado ofreciera los mejores recursos

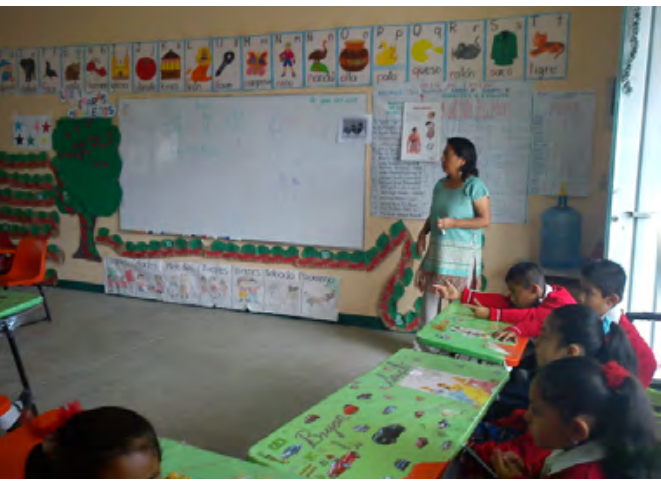
a las poblaciones más desfavorecidas, ha tendido a concentrar las mejores condiciones en las zonas urbanas, donde en efecto hay más estudiantes, pero también es donde los niños y niñas tienen más oportunidades extraescolares para aprender. En cambio, en el campo, en las localidades pequeñas y dispersas y en las comunidades indígenas, es donde hay las mayores carencias educativas, incluso en algunas regiones no hay ni siquiera escuelas, por lo que los niños tienen que caminar diariamente a otras localidades para poder estudiar. Mostremos tres gráficas para apreciar estas diferencias.

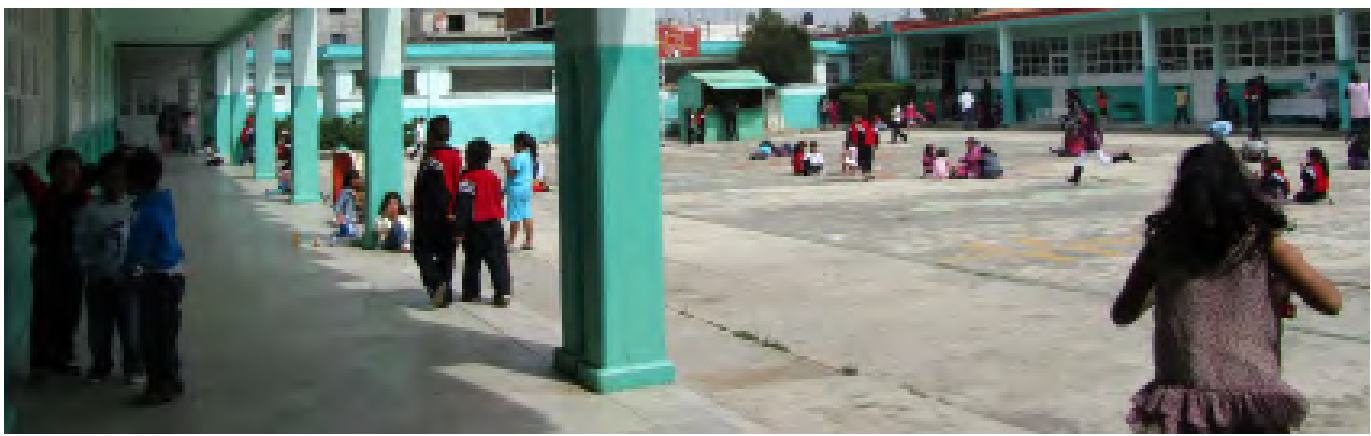


Gráfica 1. Porcentaje de grupos de $4^{\circ}, 5^{\circ}$ y $6^{\circ}$ de primaria donde todos los estudiantes tienen mueble en buenas o regulares condiciones para sentarse y escribir: nacional y por tipo de escuela

Distribución porcentual de grupos donde todos los estudiantes tienen mueble en buenas o regulares condiciones para sentarse y escribir: nacional y por tipo de escuela

Informante: Docente/LEC

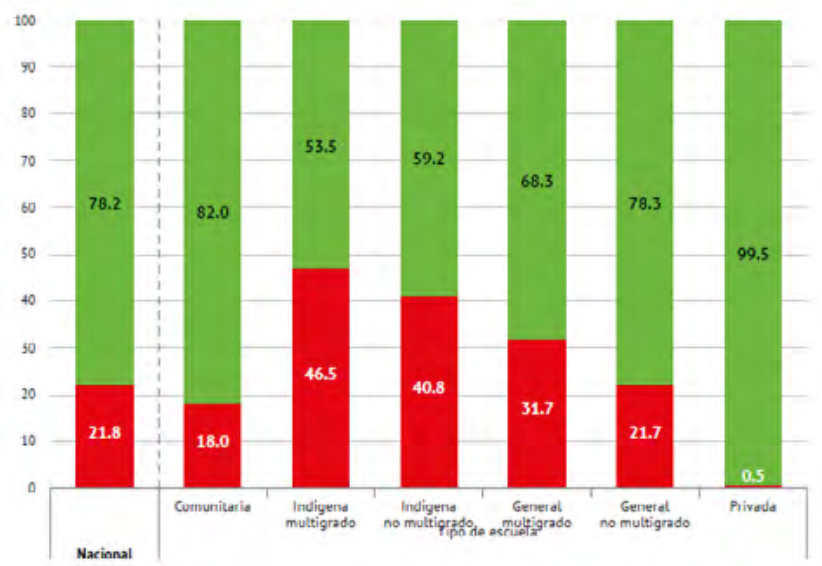

Gráfica 2. Porcentaje de grupos de $4^{\circ}, 5^{\circ}$ y $6^{\circ}$ de primaria según la proporción de estudiantes que cuenta con un juego completo de libros de texto: nacional y por tipo de escuela

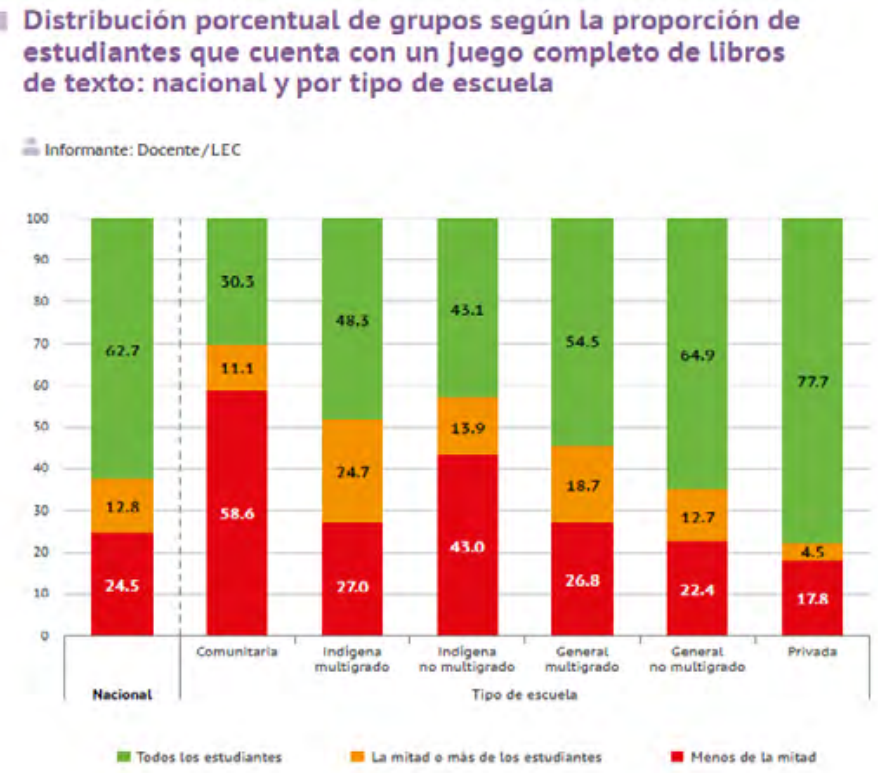


9. Psicólogo, terapeuta del lenguaje, especialista en discapacidad, asesor pedagógico, apoyo de USAER

10. Se excluyen de la gráfica las escuelas Comunitarias

Fuente: INEE (2016c)

\section{Gráfica 3. Porcentaje de escuelas que reportaron estudiantes con disca- pacidad o necesidades educativas especiales y escuelas donde se informó que se cuenta con apoyo de personal especializado ${ }^{9}$ para su atención: nacional y por tipo de escuela ${ }^{10}$}

Distribución porcentual de escuelas donde se reportaron estudiantes con discapacidad o NEE y escuelas donde se informó que se cuenta con apoyo de personal especializado ${ }^{1}$ para su atención: nacional y por tipo de escuela ${ }^{2}$

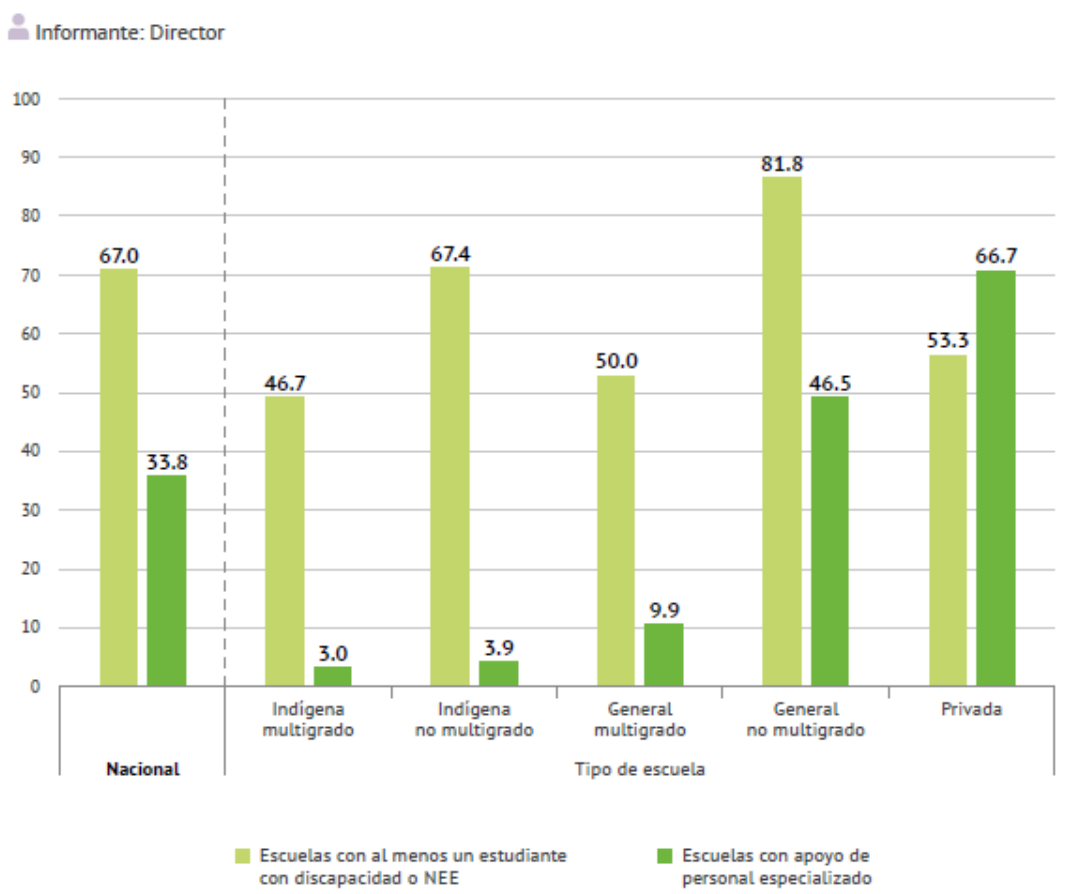

Las gráficas anteriores permiten notar que las mayores carencias de condiciones escolares están en las escuelas comunitarias, indígenas y generales multigrado, y que las brechas entre los distintos tipos de servicio son amplias, lo que muestra la manera tan desigual con que opera la educación en nuestro país.

Con la finalidad de orientar políticas educativas que aseguren un "piso mínimo común" de oportunidades educativas para todos los estudiantes del país, el Instituto Nacional para la Evaluación de la Educación (INEE) diseñó la Evaluación de condiciones básicas para la enseñanza y el aprendizaje (ECEA), la cual utiliza como referente un marco básico de condiciones sobre cómo y con qué deben funcionar mínimamente todas las escuelas en México, con base principalmente en lo que la misma normatividad y la política educativa establecen. Este marco básico está conformado por siete ámbitos y 21 dimensiones (ver Tabla 1). 
11. Una descripción muy sucinta de lo que evalúa la ECEA se puede encontrar en: http://www.inee. edu.mx/images/stories/2015/ ecea2015/ECEA21201.pdf.

\section{Tabla 1. Ámbitos y dimensiones que incluye la ECEA ${ }^{11}$}

\begin{tabular}{|c|c|}
\hline Ámbitos & Dimensiones \\
\hline $\begin{array}{l}\text { Infraestructura para el } \\
\text { bienestar y aprendizaje } \\
\text { de los estudiantes }\end{array}$ & $\begin{array}{l}\text { - Servicios básicos en el plantel } \\
\text { - Espacios escolares suficientes y accesibles } \\
\text { - Condiciones básicas de seguridad e higiene }\end{array}$ \\
\hline $\begin{array}{l}\text { Mobiliario y equipo } \\
\text { básico para la } \\
\text { enseñanza y el } \\
\text { aprendizaje }\end{array}$ & $\begin{array}{l}\text { - Mobiliario suficiente y adecuado } \\
\text { - Equipamiento de apoyo para la enseñanza y el aprendizaje }\end{array}$ \\
\hline $\begin{array}{l}\text { Materiales de apoyo } \\
\text { educativo }\end{array}$ & $\begin{array}{l}\text { - Materiales curriculares existentes (plan y programas de estudio, libros } \\
\text { de texto gratuito, libros para el maestro) } \\
\text { Materiales didácticos existentes (materiales de apoyo para la } \\
\text { implementación del currículo, acervos bibliográficos escolares y de } \\
\text { aula) }\end{array}$ \\
\hline $\begin{array}{l}\text { Personal que labora en } \\
\text { las escuelas }\end{array}$ & $\begin{array}{l}\text { - Perfil profesional de directivos y docentes de acuerdo a su función } \\
\text { - } \quad \text { Personal suficiente y que permanece durante el ciclo escolar } \\
\text { - Oportunidades de actualización profesional en la escuela }\end{array}$ \\
\hline Gestión del aprendizaje & $\begin{array}{l}\text { - Uso efectivo del tiempo para la implementación del currículo } \\
\text { - } \\
\text { - Esáctica docente orientada al aprendizaje } \\
\text { estudiantes }\end{array}$ \\
\hline Organización escolar & 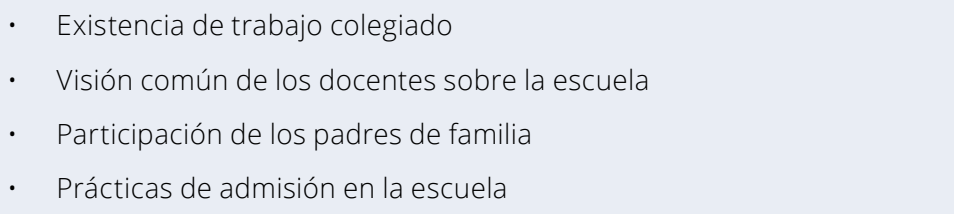 \\
\hline $\begin{array}{l}\text { Convivencia escolar } \\
\text { para el desarrollo } \\
\text { personal y social }\end{array}$ & $\begin{array}{l}\text { - Relaciones interpersonales positivas } \\
\text { - } \text { manécticas de disciplina que respetan los derechos de los estudiantes y } \\
\text { - Participación activa de los estudiantes } \\
\text { - Prácticas de inclusión hacia los estudiantes de la escuela }\end{array}$ \\
\hline
\end{tabular}

Los cuatro primeros ámbitos se refieren a recursos que, en el caso de las escuelas públicas del país, el Estado provee, es decir, corresponde a la autoridad educativa la responsabilidad de asegurar su cumplimiento. Los últimos tres ámbitos son procesos cuya responsabilidad principal recae en las propias comunidades escolares, aunque se reconoce que existe una interrelación entre ellos (por ejemplo, si la escuela no cuenta con espacios físicos para el trabajo colaborativo entre docentes, que es una condición de infraestructura, se afectará el trabajo colegiado, que alude a una condición de organización escolar). 


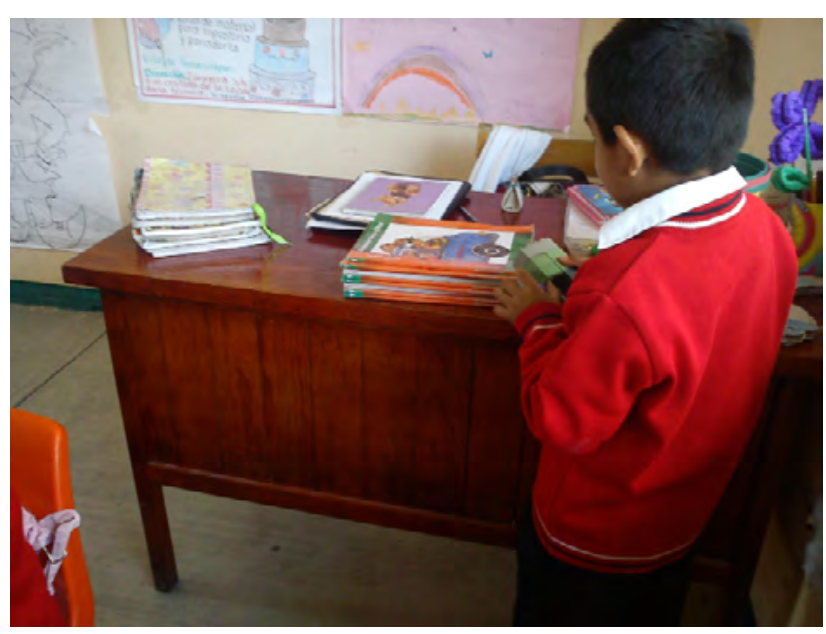

Al referirse a condiciones básicas o mínimas, el marco básico de la ECEA es indivisible, lo que significa que debe asegurarse el cumplimiento de todas y no sólo de algunas de las condiciones.

Si el lector quiere conocer más de esta evaluación lo invitamos a entrar al micrositio: http://www.inee.edu.mx/index. php/proyectos/ecea, así como ver este video:

\section{Reflexiones finales}

El derecho a la educación es más que el acceso y disponibilidad de servicios educativos; incluye la calidad de las condiciones y servicios con que se ofrecen. Ir a la escuela es necesario, pero no suficiente para el ejercicio pleno de este derecho, que implica en última instancia el derecho a aprender cosas relevantes para la vida.

En general en México, las escuelas en los contextos más pobres son las que tienen condiciones más precarias y ofrecen menor bienestar y oportunidades de aprendizaje a sus estudiantes; a estas escuelas no sólo no se les provee de mayores apoyos para subsanar las desventajas sociales, sino que ni siquiera se les da lo mismo que a otras ubicadas en contextos más favorables. Esto muestra la inequidad y la desigualdad en la oferta educativa.

Las escuelas deben ofrecer igualdad de oportunidades para todos los estudiantes, cuando menos deben asegurar condiciones básicas para funcionar. Las condiciones básicas son "irreductibles", y por tanto necesarias para la enseñanza y el aprendizaje; por ello deben atenderse en conjunto y para todas las escuelas. De lo contrario el derecho a la educación seguirá siendo un privilegio de algunos y no un derecho de todos.

\section{Referencias}

* Comisión Nacional de Derechos Humanos (2016). Los principios de universalidad, interdependencia, indivisibilidad y progresividad de los derechos humanos. México: autor. Disponible en: http://www.cndh.org.mx/sites/all/doc/cartillas/2015-2016/34Principios-universalidad.pdf 
- INEE (2016a). Panorama Educativo de México 2015. Indicadores del Sistema Educativo Nacional. Educación básica y media superior. México: INEE. Disponible en: http:// publicaciones.inee.edu.mx/buscadorPub/P1/B/114/P1B114.pdf.

- INEE (2016b). Directrices para mejorar la atención educativa de niñas, niños y adolescentes de familias de jornaleros agrícolas migrantes. México: INEE. Disponible en: http://publicaciones.inee.edu.mx/buscadorPub/P1/F/103/P1F103.pdf.

- INEE (2016c). Reporte general de resultados de la Evaluación de Condiciones Básicas para la Enseñanza y el Aprendizaje (ECEA) 2014 / Primaria. México: INEE. Disponible en: http://www.inee.edu.mx/images/stories/2016/ecea/resultadosECEA-2014actualizacion.pdf.

- INEE (2016d). Infraestructura, mobiliario y materiales de apoyo educativo en las escuelas primarias. ECEA 2014. México: INEE. Disponible en: http://publicaciones.inee. edu.mx/buscadorPub/P1/D/244/P1D244.pdf.

- INEE (2016e). La Educación Obligatoria en México. Informe 2016. Capítulo 2: Condiciones escolares para la enseñanza y el aprendizaje en la educación primaria. México: INEE. Disponible en: http://publicaciones.inee.edu.mx/buscadorPub/P1///241/ P1/241.pdf.

- INEE (2016f). Evaluación de Condiciones Básicas para la Enseñanza y el Aprendizaje desde la perspectiva de los derechos humanos. Documento conceptual y metodológico. México: INEE. Disponible en: http://publicaciones.inee.edu.mx/buscadorPub/ P1/E/201/P1E201.pdf.

- INEE (2017a). Principales cifras. Educación básica y media superior. Inicio del ciclo escolar 2015-2016. México: INEE. Disponible en: http://publicaciones.inee.edu.mx/ buscadorPub/P2/M/108/P2M108.pdf

- INEE (2017b). La educación obligatoria en México. Informe 2017. Capítulo 3: Oportunidades educativas de niñas, niños y adolescentes con discapacidad. México: INEE. Disponible en: http://publicaciones.inee.edu.mx/buscadorPub/P1///242/P1/242.pdf.

- INEE (2017c). Breve panorama educativo de la población indígena. Día Internacional de los Pueblos Indígenas. México: INEE. Disponible en: http://publicaciones.inee. edu.mx/buscadorPub/P3/B/107/P3B107.pdf.

* Latapí, P. (2009). El derecho a la educación: su alcance, exigibilidad y relevancia para la política educativa. Revista Mexicana de Investigación Educativa, 14(40). Disponible en: http://www.comie.org.mx/v1/revista/portal.php?idm=es\&sec=SC03\& \&sub=SBB\&criterio=ART40012.

* Tomasevski, K. (2004). Indicadores del derecho a la educación. Revista IIDH, 40. Disponible en: https://revistas-colaboracion.juridicas.unam.mx/index.php/revinstituto-interamericano-dh/article/view/8220/7368

* Vázquez, L. D. y Serrano, S. (s/f). Los principios de universalidad, interdependencia, indivisibilidad y progresividad. Apuntes para su aplicación práctica. Disponible en: https://archivos.juridicas.unam.mx/www/bjv/libros/7/3033/7.pdf 


\section{Cómo citar este artículo}

* Ahuja Sánchez, Raquel (2017). ¿Qué tan derecho es el derecho a la educación en México?, Revista Digital Universitaria (RDU), vol. 18, núm. 7, septiembre-octubre. Recuperado de http://dx.doi.org/10.22201/codeic.16076079e.2017.v18n7.a1. 